\title{
Transformation Model and Constraints Cause Bias in Statistics on Deformation Fields
}

\author{
Torsten Rohlfing \\ Neuroscience Program, SRI International, Menlo Park, CA, USA \\ torsten@synapse.sri.com \\ http://www.stanford.edu/ rohlfing/
}

\begin{abstract}
This work investigates the effects of nonrigid transformation model and deformation constraints on the results of deformation-based morphometry (DBM) studies. We evaluate three popular registration algorithms: a B-spline algorithm with several different constraint terms, Thirion's demons algorithm, and a curvature PDE-based algorithm. All algorithms produced virtually identical overlaps of corresponding structures, but the underlying deformation fields were very different, and the Jacobian determinant values within homogeneous structures varied dramatically. In several cases, we observed bi-modal distributions of Jacobians within a region that violate the assumption of gaussianity that underlies many statistical tests. Our results demonstrate that, even with perfect overlap of corresponding structures, the statistics of Jacobian values are affected by bias due to design elements of the particular nonrigid registration. These findings are not limited to DBM, but also apply to voxel-based morphometry to the extent that it includes a Jacobian-based correction step ("modulation").
\end{abstract}

\section{Introduction}

Deformation-based morphometry (DBM) [1,2] is an increasingly popular method to assess anatomical population differences between different groups [3], or to track changes in individual subjects over time [4]. Both changes over time and differences between groups are commonly represented and analyzed as the Jacobian determinant maps of nonrigid coordinate transformations between the images involved [5]. This procedure is based on the fact that the Jacobian of the transformation at each point represents the local volume change of the coordinate space.

There is a fundamental difference between the application of nonrigid registration for the purpose of matching two images and for DBM. For multi-modality image fusion, for example, the sole criterion for success is the correct match of visible image features. However, when performing analysis of the deformation itself, as is done in DBM, the information stored in the deformation does not depend on the image content alone. We can, therefore, no longer rely on feature alignment. Two different deformation fields may achieve equal accuracy at all visible features, yet be very different in regions without such features. Ideally, the deformation should be equally distributed inside homogeneous structures. In the brain, for example, if there is a volume change in the ventricles, then the deformation should reflect this homogeneously rather than have substantial peaks in some areas. 
A common view of image registration is that it is driven by two types of forces: 1) external forces that result from the dissimilarity of the two images that are being matched and that drive the transformation in the direction that improves the image match, and 2) internal forces that aim to minimize an inherent energy of the coordinate mapping, thus for example enforcing its smoothness. In homogeneous image regions, the image forces should be zero, because the image similarity measure is only sensitive to changes in the transformation that lead to a change in mapped pixel intensities. In such regions, the transformation is determined entirely by the internal forces. Our requirement as stated above thus translates into the condition that the internal forces alone should lead to a distribution of local volume changes that is maximally uniform.

On a conceptual level, a nonrigid registration method is defined by 1) an optimization method, 2) an image similarity metric, 3 ) a parametric coordinate transformation model, and, finally, 4) a regularizer or smoother that favors legitimate transformations over such that are not physically or otherwise reasonable. From these four building blocks, we can assume that the optimal similarity measure is determined by the types of images to be registered. The optimal registration transformation in regions with no image forces is then exclusively determined by transformation model and regularizer. We, therefore, focus in this paper on evaluating the effects that these two components have on the statistics of Jacobian determinants as they are commonly used in DBM.

\section{Methods}

We evaluate in this work three popular examples of nonrigid registration algorithms, for which implementations are publicly available. The particular selection of algorithms does not imply any judgment of the performance of these compared with other methods, except for our group's experience that each of the three method below is effective at successfully registering both intra-subject (i.e., longitudinal) and inter-subject images.

\subsection{B-Spline Registration}

Rueckert et al. [6] introduced a nonrigid registration algorithm that uses B-spline interpolation between uniformly spaced control points as its transformation model. The control points move independently, thus deforming the underlying image, until a local maximum of the image similarity measure is found. We use an algorithm similar to Rueckert's, but, for the purpose of this study, the sum of squared differences (SSD) image similarity measure is used rather than normalized mutual information.

The B-spline transformation model incorporates implicit smoothness and continuity constraints. Nonetheless, it was recognized early that additional constraints can be necessary to enforce physically meaningful transformations, and to incorporate prior information about permissible deformations. This is achieved by adding a weighted constraint term $E_{\text {constraint }}$ (see below) to the similarity measure. We thus obtain the total cost function $E_{\text {total }}=E_{\mathrm{SSD}}+\omega E_{\text {constraint }}$, which is minimized during the registration. In this paper, we evaluate the following regularization approaches:

1. Unconstrained registration, driven only by the similarity measure, which is equivalent to a zero constraint term. 
2. Smoothness constraint based on second-order derivatives of the transformation that models the bending energy of a thin metal plate [6]7]. For details on the computation of the constraint using derivatives of the B-spline transformation, see [8].

3. Volume preservation constraint as introduced by Rohlfing et al. [8]. The constraint penalizes the deviation of the local Jacobian from 1 (or, more generally, the global affine scaling factor) anywhere in the image. For small weights $\omega$, the volume preservation constraint is relaxed and becomes a folding prevention constraint.

\subsection{Demons Registration}

Thirion [9] introduced a registration algorithm based on image forces and Gaussian regularization of the deformation field. The transformation model of this method is a local displacement field: at each and every pixel $\mathbf{x}$ the deformation is described by a displacement vector $\mathbf{u}[\mathbf{x}]$ such that $\mathbf{T}[\mathbf{x}]=\mathbf{x}+\mathbf{u}[\mathbf{x}]$. The algorithm updates the displacement field $\mathbf{u}$ iteratively. The field is updated in each step by adding a delta in the direction of the image force field to the displacement field from the previous step. For regularization, convolution with a Gaussian kernel smooths the updated deformation field after each step. The image forces for the registrations in this paper are computed by a symmetric force based on both reference and sample image gradients as suggested by [9]. We use an implementation of the algorithm that is publicly available as part of the Insight Toolkit (ITK).

\subsection{Curvature-Based Registration}

Variational registration algorithms casts the registration problem as a system of differential equations and solves these using variational methods. Popular examples of variational methods include elastic matching [10] and viscous fluid registration algorithms [11]. The transformation model for variational methods is a local displacement field, analogous to one used by the demons algorithm. The registration is driven by two components, a distance function $D$ that is minimized when both images are identical, and a regularization term (also known as smoother) $S$ that is minimized when the deformation field satisfies some smoothness constraints. The registration problem thus becomes the problem of minimizing $E_{\text {total }}=D[R, T ; \mathbf{u}]+\alpha S[\mathbf{u}]$, that is, we need to minimize the image distance $D$ while simultaneously minimizing the smoothing term $S$, weighted by an adjustable parameter $\alpha$. In this paper in particular, we use the curvature regularization term introduced by Fischer \& Modersitzki [12]. This term has several advantageous properties: it does not penalize global affine transformation components, and it can be implemented with time complexity $O(n \log n)$, where $n$ is the number of image pixels. We have made our implementation of this algorithm publicly available by contributing it to ITK. For the purpose of this paper, the image forces that drive the registration are implemented as the symmetric demons forces as described by [9].

\subsection{Electronic Phantom}

We model volume gain and volume loss using a pair of simple geometric phantom images, depicted in Fig. 1. Each of the two images has $128^{3}$ isotropic voxels and contains 


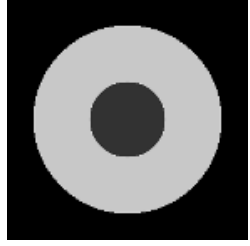

(a) Inner radius 40 pixels

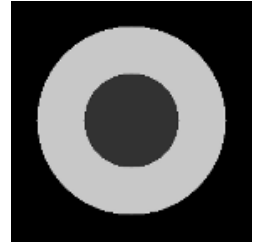

(b) Inner radius 50 pixels

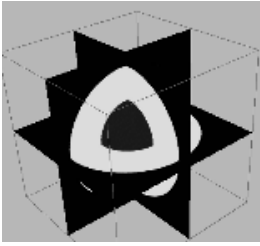

(c) Triplanar slices

Fig. 1. Electronic phantom images. The pictures show the central axial slices through the isotropic 3D volume. Due to spherical symmetry, the other orthogonal central slices are identical. The radius of the outer sphere is 100 pixels in both images. The radius of the inner sphere is 40 pixels in image (a), and 50 pixels in image (b). Picture (c) shows a three-dimensional rendering of triplanar slices through the volumetric phantom image with 50 pixel inner sphere.

two concentric spheres. The radius of the outer sphere is 100 voxels, whereas the radius of the inner sphere is 40 voxels in one image, and 50 voxels in the other. The voxels inside the inner spheres have constant pixel intensity 50, the voxels inside the outer sphere but outside the inner sphere have constant intensity 200. The image background outside the outer sphere is set to zero. One can think of these images as extremely crude approximations of the human brain and the ventricles. Note that a key characteristic of our phantom images, due to the constant size of the outer sphere, is that they cannot be registered using a global affine transformation, i.e., we force a successful registration transformation to be truly nonrigid.

The volumes (in pixels) of the inner spheres in the two images are $V_{i, 40}=267761$ for thew sphere with radius 40 pixels, and $V_{i, 50}=523305$ for the sphere with radius 50 pixels. The volumes of the outer spheres after subtraction of the inner spheres' volumes are $V_{o, 40}=3920096$ and $V_{o, 50}=3664552$. Based on these volumes, we can compute the "ideal" Jacobian determinants $J_{i}$ and $J_{o}$ of the deformation field throughout the inner and outer sphere. For registration using the 40-pixel inner sphere as the reference image, these factors are $J_{i}=V_{i, 50} / V_{i, 40} \approx 1.954$ and $J_{o}=V_{o, 50} / V_{o, 40} \approx 0.935$.

\section{Results}

All registration methods performed virtually indistinguishable as judged by achieving overlap between corresponding structures in the two images (recognition rates between 0.9994 and 0.9997 for all methods). The residual differences were caused by sub-pixel misalignments along the boundaries of the different structures, which was verified by visually inspecting the subtraction images.

The results for the demons and curvature registration algorithms are visualized in Fig. 2. The results for the B-spline registration algorithm are visualized in Fig. 3 . For each algorithm, the images on the left visualize the deformation field as gray-level encoded Jacobian values (left half) and as deformed Cartesian grids (right half). These serve to assess the spatial structure of the variation within the deformation field. The two line graphs show the distribution of Jacobians over all pixels in the inner (left) and outer (right) sphere. The average Jacobians in these graphs are represented by 

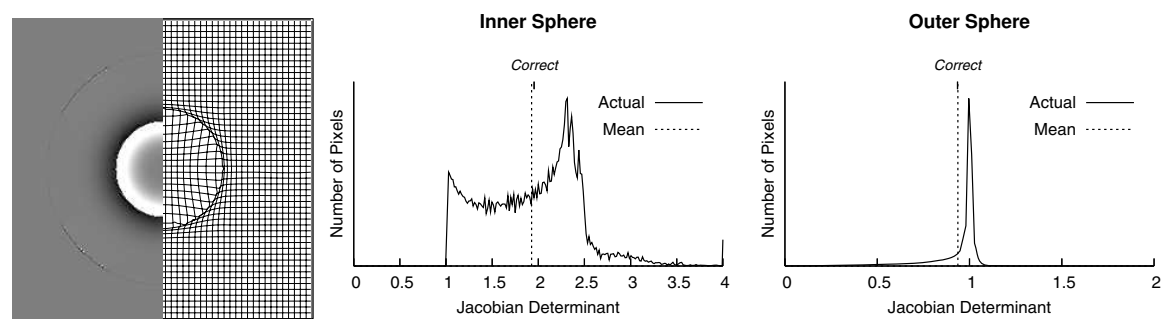

(a) Demons algorithm, single resolution
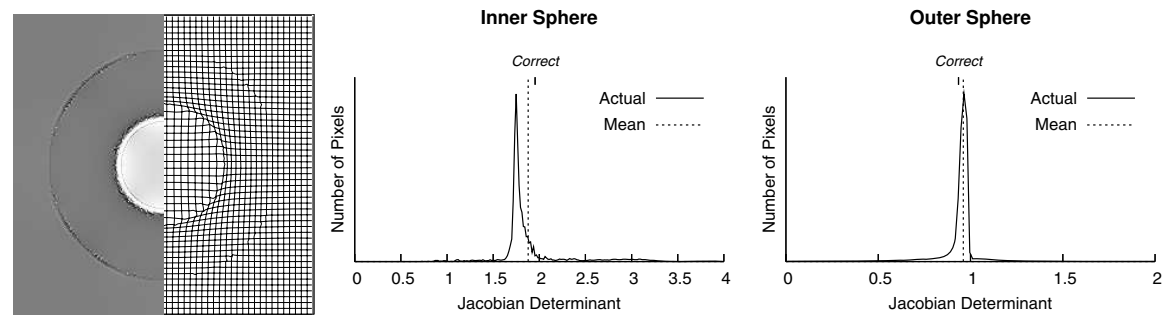

(b) Demons algorithm, multi resolution
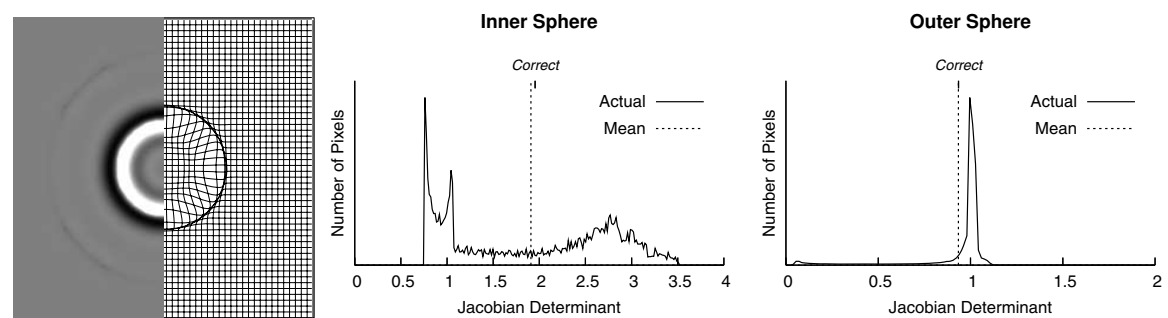

(c) Curvature registration, single resolution
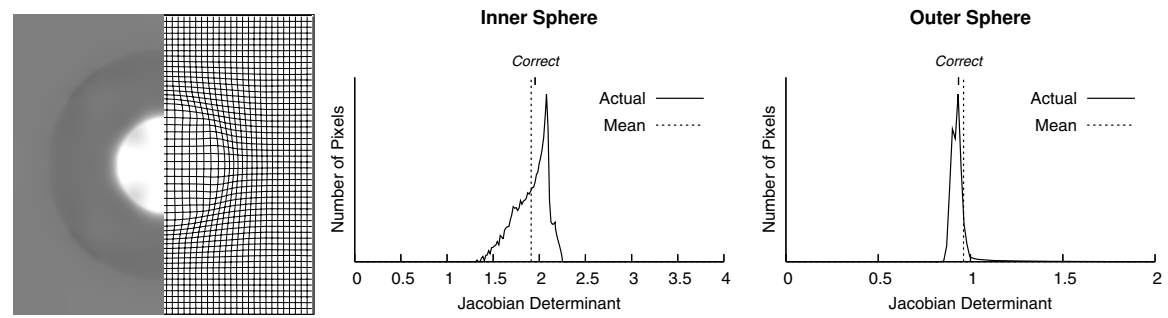

(d) Curvature registration, multi resolution

Fig. 2. Results for PDE-based registration methods. For each method we show (from left to right): Jacobian map of the coordinate transformation; deformed Cartesian grid; plots of the distributions of Jacobians for the inner and outer spheres. Note that Jacobian map and deformed grid are essentially symmetric with respect to the image center, and only one half of each is shown. Note also that the deformed grid is represented in the coordinates of the target image and thus does not match the structure of the Jacobian map, which is represented in the coordinates of the reference image. 

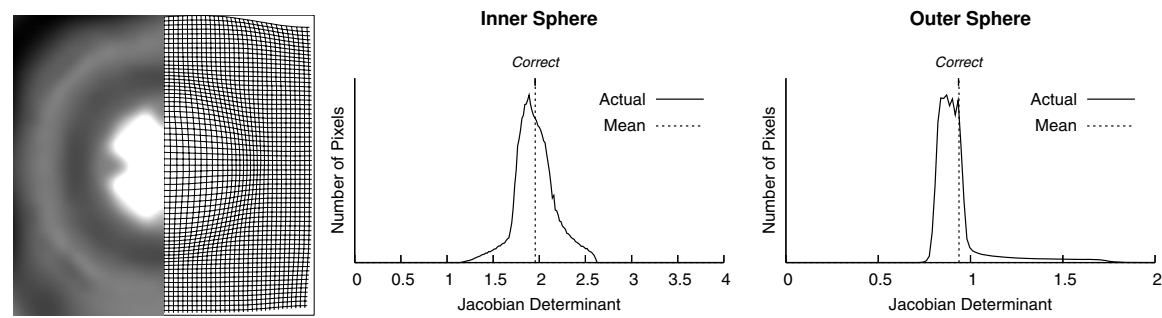

(a) B-spline registration, unconstrained
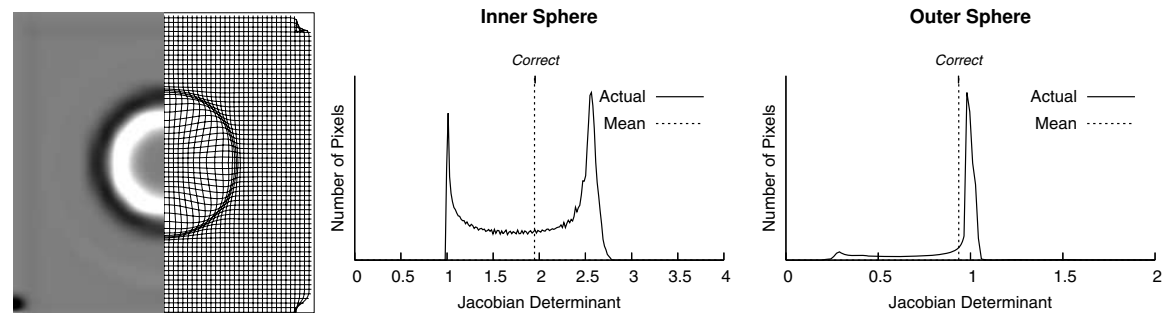

(b) B-spline registration, smoothness constraint $\left(\omega=5 \cdot 10^{-1}\right)$
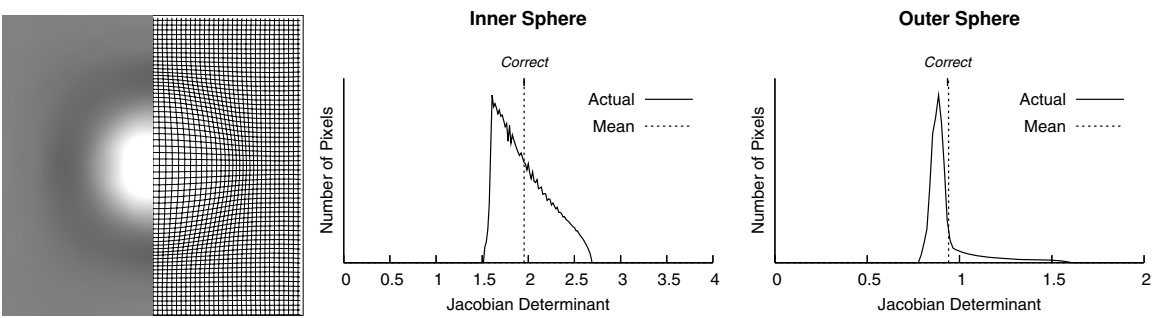

(c) B-spline registration, volume-preservation constraint $\left(\omega=10^{-3}\right)$

Fig. 3. Results for the B-spline registration method with different deformation constraint terms. See text and caption for Fig. 2 for details.

dashed vertical lines. Ticks labeled "Correct" mark the a priori correct values. All Jacobian maps are shown with a linear gray scale centered at 1.0 with a window width of 2.0.

All registration methods produced deformations fields for which the average Jacobian over each region was very close to the theoretically correct value. This is a necessary side effect of the correctly compute volume overlap. The multi-resolution demons and curvature algorithms approximated the correct value for the smaller sphere with slightly lower accuracy than the other method, but only marginally so.

The unconstrained B-spline registration (Fig.3.a)) produced a deformation field with a noticeable geometric structure, which is determined by the properties of the B-spline. This observation, which is also illustrated in Fig. 4, previously appeared in graphical form in works by others (e.g., [5]), but was never considered in detail as a fundamental issue for subsequent deformation field analysis. 


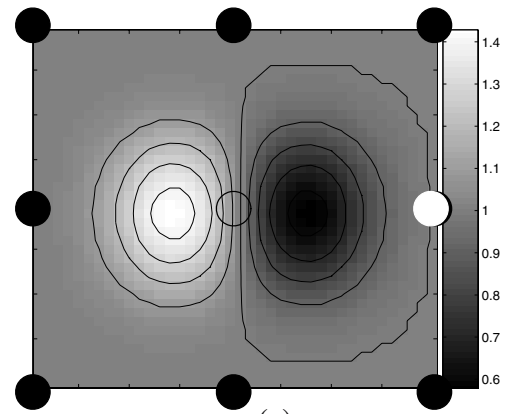

(a)

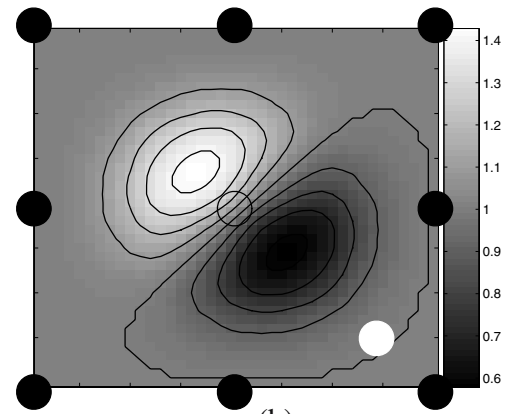

(b)

Fig. 4. Jacobian determinant map of B-spline free-form deformations with different angles of control point movement. Each image is covered by $3 \times 3$ control points (solid circles). (a) Central control point (empty circle) moved by $(+1,0)$ grid units (to solid white circle). (b) Central control point moved by $\left(+\frac{1}{\sqrt{2}},+\frac{1}{\sqrt{2}}\right)$. Note the different shapes of the two iso-Jacobian contour sets.

Noteworthy in Fig.2 1 is the improved spatial homogeneity of the Jacobian maps when a multi-resolution strategy (4 levels) is used. However, the multi-resolution strategy introduces geometrical artifacts similar to those observed in Fig. 3. a) for the unconstrained B-spline registration. Here, this effect is an artifact of refining coarser deformation fields (with larger voxels) to finer resolutions.

None of the registration algorithms produced either uniform (desirable from a conceptional point of view) or Gaussian (desirable for statistical testing) distributions of Jacobians. Several registration algorithms even produced distributions in the smaller sphere that were bi-modal: B-spline with smoothness constraint $\left(\omega=5 \cdot 10^{-1} ;\right.$ Fig. 3 b $)$ ), and the single-resolution demons and curvature algorithms (Fig. 22(a) and (c)). Such distributions grossly violate the assumption of Gaussian distribution of the Jacobian values that is made by most commonly applied statistical tests, e.g., t-tests.

\section{Discussion}

The key concept in DBM is the pixelwise comparison and statistical testing of measures derived from nonrigid coordinate transformations. The results presented in this paper demonstrate how such derived measures are determined by the absence of image features, and by the mathematical properties of the nonrigid coordinate transformation model. This includes any regularizers used to constrain the optimization problem. These effects are not random, but show strong spatial and distributional structure. DBM results may, therefore, vary greatly depending on the particular choice of the building blocks of the algorithm that is used for registration.

None of the methods tested in this work performed perfectly, although the multiresolution demons algorithm appeared to have the relatively lowest level of artifact. Nonetheless, we found that the demons algorithm, too, produced a bi-modal, and thus highly nongaussian, Jacobian distribution when run at only a single spatial resolution. On the positive side, because for all methods the average of the Jacobian determinants 
over all pixels in homogeneous regions was close to the correct volume ratios, we suggest that DBM best be performed by segmented regions rather than locally by pixels.

We note that the problems described in this paper are not limited to DBM, but also apply to application of voxel-based morphometry (VBM) [13]. The so-called "optimized VBM" [14] protocol incorporates a "modulation" step, in which the maps of brain tissue weights are corrected for inter-subject volume changes. This correction is achieved by multiplying the tissue weights at each pixel with the Jacobian determinant of the transformation that maps each subject into template space.

\section{Acknowledgments}

This work was supported by the National Institute on Alcohol Abuse and Alcoholism, Grants No. AA05965, AA10723, AA12388, AA12999, AA13521, and the National Institute on Aging, Grant No. AG17919. Many helpful comments and suggestions from A. Pfefferbaum, E.V. Sullivan, and K. Pohl are greatly appreciated.

\section{References}

1. Ashburner, J., Hutton, C., Frackowiak, et al.: Identifying global anatomical differences: Deformation-based morphometry. Hum. Brain Map. 6(5-6) (1998) 348-357

2. Csernansky, J.G., Joshi, S., Wang, L., et al.: Hippocampal morphometry in schizophrenia by high dimensional brain mapping. Proc. Natl. Acad. Sci. USA 95(19) (1998) 11406-11411

3. Gaser, C., Volz, H.P., Kiebel, S., et al.: Detecting structural changes in whole brain based on nonlinear deformations — application to schizophrenia research. NeuroImage 10(2) (1999) $107-113$

4. Shen, D., Davatzikos, C.: Very high-resolution morphometry using mass-preserving deformations and HAMMER elastic registration. NeuroImage 18(1) (2003) 28-41

5. Chung, M.K., Worsley, K.J., Paus, T., et al.: A unified statistical approach to deformationbased morphometry. NeuroImage 14(3) (2001) 595-606

6. Rueckert, D., Sonoda, L.I., Hayes, C., et al.: Nonrigid registration using free-form deformations: Application to breast MR images. IEEE Trans. Med. Imag. 18(8) (1999) 712-721

7. Wahba, G.: Spline Models for Observational Data. Volume 59 of CBMS-NSF Regional Conference Series. Society for Industrial and Applied Mathematics, Philadelphia, PA (1990)

8. Rohlfing, T., Maurer, Jr., C.R., Bluemke, D.A., et al.: Volume-preserving nonrigid registration of MR breast images using free-form deformation with an incompressibility constraint. IEEE Trans. Med. Imag. 22(6) (2003) 730-741

9. Thirion, J.P.: Fast non-rigid matching of 3D medical images. Technical Report 2547, INRIA, Sophia Antipolis, France (1995)

10. Bajcsy, R., Kovačič, S.: Multiresolution elastic matching. Comput. Vision. Graph. Image Processing 46(1) (1989) 1-21

11. Christensen, G.E., Rabbitt, R.D., Miller, M.I.: Deformable templates using large deformation kinematics. IEEE Trans. Image Processing 5(10) (1996) 1435-1447

12. Fischer, B., Modersitzki, J.: Curvature based image registration. J. Mathematical Imaging and Vision 18(1) (2003) 81-85

13. Ashburner, J., Friston, K.J.: Voxel-based morphometry - the methods. NeuroImage 11(6) (2000) 805-821

14. Good, C.D., Johnsrude, I.S., Ashburner, J., et al.: A voxel-based morphometric study of ageing in 465 normal adult human brains. NeuroImage 14(1) (2001) 21-36 